\title{
Multiple extracranial metastases from intradiploic meningioma
}

Sir,

We report a case of a 46-year-old lady who presented with $15 \times 15 \mathrm{~cm}$ swelling in the left gluteal region with radiating pain, weakness in her left lower limb, diminished perianal sensations and absent ankle jerks. Three years earlier she had undergone a subtotal excision of a left parietal intradiploic fibroblastic meningioma (MIB-1 index of 1\%). It was attached to the underlying superior sagittal sinus, from which it was shaved off. A computed tomogram (CT) of the brain one year post-subtotal excision showed a $1 \mathrm{~cm}$ residual lesion in the left parietal parasagittal region for which she was advised a repeat surgery. She declined to have surgery. At the current presentation X-ray of the pelvis showed a lytic lesion involving the entire left side of the sacrum. CT of the brain showed a 3 -cm enhancing mass at the site of previous surgery (left parietal parasagittal region) [Figure 1]. CT scan of the sacrum showed an $11 \times 11 \times 9 \mathrm{~cm}$ enhancing soft tissue mass destroying the sacrum, coccyx and iliac aspect of the left sacroiliac joint, infiltrating surrounding tissues and vertebral canal [Figure 2]. Angiography of the iliac vessels showed a tumor blush in the sacral region with feeders from both the internal and right external iliac arteries. X-ray cervical spine showed anterolisthesis of C2 on C3 with lucent areas in the body and lamina of C2. Bone scan showed increased uptake of tracer in the skull, cervical spine, left scapula and sacrum.

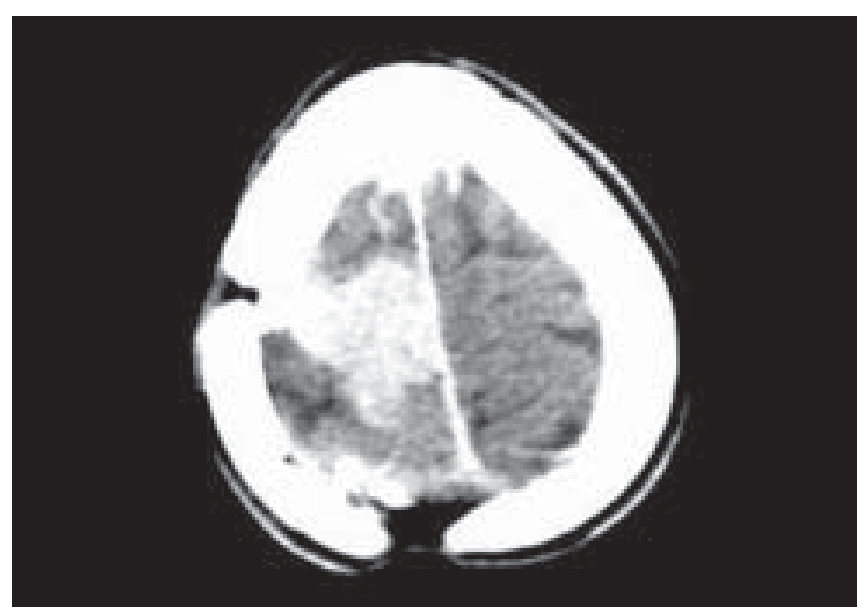

Figure 1: Contrast CT scan of the brain showing an enhancing mass in the left parietal parasagittal region 
She underwent embolization of the internal iliac branches feeding the mass, followed by low back exploration and excision of the mass. At surgery it was found that the sacrum was destroyed. There was a dural tear that was repaired. On histopathological examination the tumor was composed of polygonal cells with oval nuclei and eosinophilic cytoplasm exhibiting brisk mitotic activity (30-40/10 hpf). The tumor cells were strongly positive for epithelial membrane antigen and vimentin and negative for cytokeratin, S-100 protein, smooth muscle actin, CD31, CD99, GFAP and synaptophysin. A diagnosis of malignant meningioma was made [Figure 3]. It was not possible to determine if this represented a metastatic deposit from the parasagittal mass or arose de novo although it is more likely to be the former. Following surgery she was relieved of her back pain. She developed wound infection with CSF leak, which was managed with resuturing, antibiotics and lumbar subarachnoid drain. After the wound healed she was

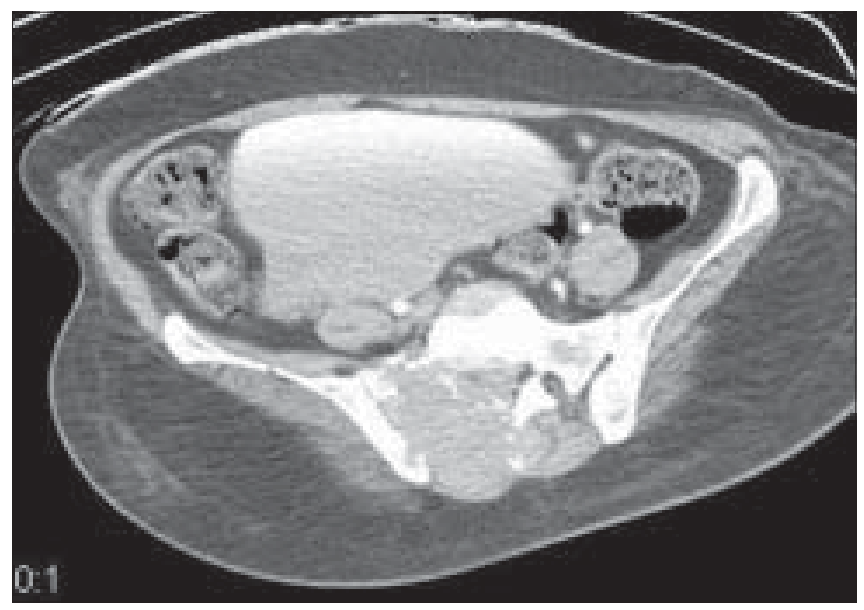

Figure 2: Contrast CT scan of the sacrum showing the mass, destruction of the sacrum and extension of the mass into the surrounding muscles

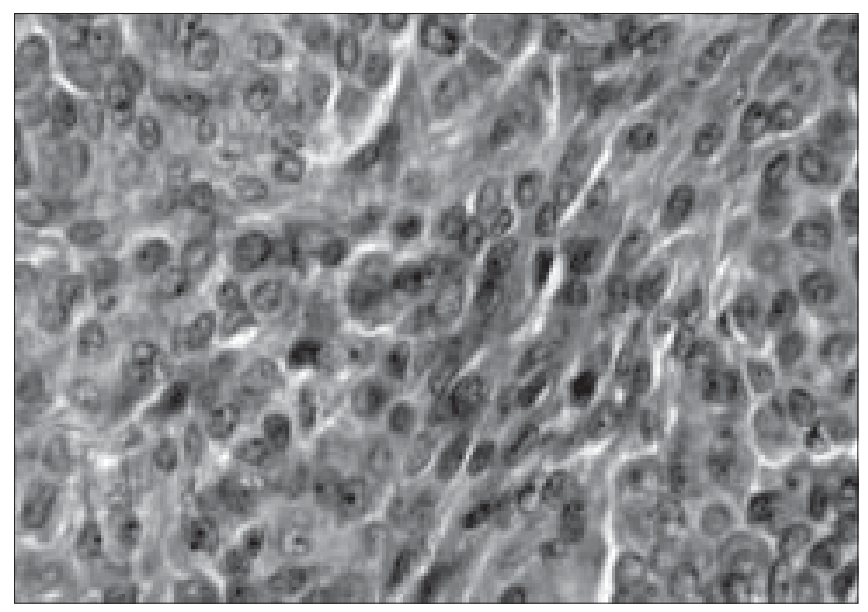

Figure 3: Photomicrograph showing the polygonal cells on histopathological staining $(\mathrm{H} \& \mathrm{E}, \times 90)$ given external radiotherapy to the lumbosacral (40 Gy in 20 fractions) and cervical regions (30 Gy in 10 fractions). After completion of radiotherapy she was advised chemotherapy with hydroxyurea.

The incidence of extracranial metastasis from intracranial meningioma is reported to be less than $1 \%{ }^{[1]}$ Metastases from meningiomas have been reported at various sites like lungs (most common site), bone, thyroid and muscle. ${ }^{[2]}$ Vertebral metastases have been reported to constitute $7 \%$ of all remote metastases from meningiomas. ${ }^{[2]}$ Meningioma metastases to the sacrum have been recently reported. ${ }^{[3]}$

The histological aggressiveness does not correlate with the metastatic potential. Benign meningiomas account for $60 \%$ of the extracranial metastases reported. ${ }^{[4]}$ Metastasis requires invasive ability and ability to grow in the new environment. Halaka et al., have demonstrated that invasive meningiomas produce collagenase and noninvasive tumors do not. ${ }^{[5]}$ The duration, site of operation and location of tumor do not seem to have a bearing on the future metastasis. Meningiomas disseminate through hematogenous, lymphatic or CSF pathways. ${ }^{[6]}$ In our case, involvement of the superior sagittal sinus found at first surgery may be the cause of both the hematogenous spread to the scapula and the rib and probable venous spread via the Batson's plexus to the $\mathrm{C} 2$ vertebra and the sacrum. Benign meningiomas that invade the venous sinus should be followed up closely as these tend to disseminate hematogenously.

\section{G. Samson Sujit Kumar, Geeta Chacko, Ari G. Chacko, \\ R. P. Haran \\ Department of Neurological Sciences, Christian Medical College and Hospital, Vellore, Tamil Nadu - 632 004, India. \\ E-mail: agchacko@cmcvellore.ac.in}

DOI: $10.4103 / 0028-3886.48801$

\section{References}

1. Strange RR, Tovi D, Nordenstam H. Meningioma with intracerebral, cerebellar and visceral metastases. J Neurosurg 1964;21:1098-102.

2. Karasick JL, Mullan SF. A survey of metastatic meningiomas. J Neurosurg 1974;40:206-12.

3. Lee YY, Wen-Wei Hsu R, Huang TJ, Hsueh S, Wang JY. Metastatic meningioma in the sacrum: A case report. Spine 2002;27:E100-3.

4. Tognetti F, Donati R, Bollini C. Metastatic spread of benign intracranial meningioma. J Neurosurg Sci 1987;31:23-7.

5. Halaka AN, Bunning RA, Bird CC, Gibson M, Reynolds JJ. Production of collagenase and inhibitor (TIMP) by intracranial tumors and dura in vitro. J Neurosurg 1983;59:461-6.

6. Ludwin SK, Conley FK. Malignant meningioma metastasizing through the cerebrospinal pathways. J Neurol Neurosurg Psychiatry 1975;38:136-42.

Accepted on 26-01-2009 